\title{
Impact and Consequences of Opioid-Induced Constipation: A Survey of Patients
}

\author{
Giustino Varrassi (D) - Vivek Banerji • Walter Gianni · Franco Marinangeli • \\ Carmine Pinto
}

Received: February 16, 2021 / Accepted: May 5, 2021 / Published online: June 3, 2021

(C) The Author(s) 2021

\section{ABSTRACT}

Introduction: Opioids are a valuable tool to help achieve control of pain. However, opioidinduced constipation (OIC) is an important limitation of treatment with this class of drugs. Methods: To better understand the impact of OIC on patient-reported outcomes, we carried out a survey involving patients being treated with opioids. Both ad hoc questions and the PROMIS and PAC-SYM and PAC-QOL scores were used.

Results: Of the 597 participants, 150 (25\%) had cancer-related pain, and 447 (75\%) had noncancer pain; 66\% experienced OIC. PROMIS pain interference questions indicated that pain

G. Varrassi $(\bowtie)$

Paolo Procacci Foundation, Via Tacito 7, 00193

Rome, Italy

e-mail: giuvarr@gmail.com

V. Banerji

Insight Dojo, Guildford, UK

W. Gianni

IRCCS Santa Lucia Foundation, Rome, Italy

F. Marinangeli

Department of MESVA, University of L'Aquila, 67100 L'Aquila, Italy

C. Pinto

Medical Oncology Unit, Clinical Cancer Center, Azienda USL-IRCCS di Reggio Emilia, Reggio Emilia, Italy is more likely to interfere with a patient's life when they have OIC. PAC-QOL and PAC-SYM revealed that $58 \%$ of patients with non-cancer pain and OIC reported at least one "severe" or "very severe" constipation symptom, compared to $83 \%$ with cancer-related pain. Younger age and less time on opioids were associated with greater impact of OIC on quality of life. Only $41 \%$ of patients were satisfied with how their constipation was managed. Over $50 \%$ of those with non-cancer pain said that they modified their opioid regimen due to constipation, vs. $6 \%$ of those with cancer pain. Constipation had been discussed with the healthcare provider (HCP) in $48 \%$ of non-cancer patients and in $73 \%$ of cancer patients. In those with chronic pain and OIC, 24\% expressed varying degrees of dissatisfaction with the healthcare system, vs. $37 \%$ in those with cancer pain and OIC.

Conclusion: Our results provide additional evidence that management of OIC is inadequate in many cases. Moreover, they indicate that there is a definite need for better education about OIC among HCPs.

Keywords: Cancer; Impact; Opioids; Opioidinduced constipation; Pain; Survey 


\section{Key Summary Points}

Why carry out this study?

Opioids are a valuable tool in the control of both cancer- and non-cancer pain, although opioid-induced constipation (OIC) is an important limitation of treatment with opioids.

A survey involving patients being treated with opioids in Italy was carried out to better understand the impact of OIC on patient-reported outcomes.

\section{What was learned from the study?}

Many patients reported that management of OIC was inadequate, thus reinforcing the need for education of healthcare providers about OIC and its negative consequences.

Prescribers should be encouraged to actively ask patients about OIC at every follow-up visit.

Overcoming barriers to inadequate management of OIC thus provides the opportunity to improve the patient's quality of life and lessen functional impairment, which is especially relevant given that effective treatment options, such as PAMORAs, are now part of a newly proposed algorithm for management of OIC.

\section{DIGITAL FEATURES}

This article is published with digital features, including a summary slide to facilitate understanding of the article. To view digital features for this article go to https://doi.org/10.6084/ m9.figshare.14402315.

\section{INTRODUCTION}

Pain remains a major public health issue of significant proportion $[1,2]$. Unfortunately, uncontrolled pain is still highly prevalent, as demonstrated in a recent study in primary care [3] and in other Italian surveys [4, 5]. The use of opioids has increased significantly in recent years to treat both cancer and non-cancer pain $[1,2,6,7]$. Indeed, opioid therapy is considered to be a valuable tool in achieving optimal control of pain in appropriately selected patients, together with adequate monitoring [1, 2]. Despite their good efficacy in controlling pain, adverse effects can often limit the long-term use of opioids, and among these opioid induced constipation (OIC) is the most frequent. A multidisciplinary group defined OIC as "a change when initiating opioid therapy from baseline bowel habits that is characterized by any of the following: reduced bowel movement frequency, development or worsening of straining to pass bowel movements, a sense of incomplete rectal evacuation, or harder stool consistency" [8]. In patients being treated with opioids, OIC may develop early and thus requires early treatment [9]. Furthermore, in cancer patients, OIC can be increased by the administration of other drugs, such as antiemetics during chemotherapy.

The exact proportion of patients experiencing OIC varies somewhat in different studies and patient populations. In a systematic review on OIC, it was reported that $41 \%$ of patients had constipation, $32 \%$ had nausea, and 29\% experienced somnolence (29\%) [10]. In a crosssectional survey of 520 patients with cancer pain, $62 \%$ of patients reported some degree of OIC, and $16 \%$ required hospitalization as a consequence [11]. In any case, it is generally accepted that roughly one-half of patients on long-term therapy with opioids will experience OIC [9], and that among those needing laxative therapy, only about $50 \%$ will achieve the desired effects [12].

In considering therapies for relief from constipation, over-the-counter laxatives as well as increased fiber and fluid intake, exercise, and stool softeners are widely used, but they are 
reported to be largely ineffective. In fact, symptoms may remain even despite the use of two or even more laxative agents, leading to inevitable negative consequences on the patients' quality of life (QoL) [13]. Prescription medications are indicated when OIC persists despite conservative approaches [14]. These include the peripherally acting m-opioid receptors antagonists (PAMORAs) [14-16]. PAMORAs are a particularly promising class of agents for OIC [17]. Among these, in addition to methylnaltrexone bromide and naloxegol, naldemedine has recently been approved for OIC. These mechanism-based treatments for OIC are valid treatment options, since they are well tolerated, do not affect analgesia, and do not cause symptoms of opioid withdrawal [17]. In Italy, methylnaltrexone bromide, naloxegol and naldemedine are all reimbursed by the National Healthcare System for OIC in adult patients on chronic opioid therapy who are resistant to at least two laxatives [18].

Unfortunately, OIC may negatively affect adherence to opioid therapy, and the symptoms of OIC may lead patients to decrease the dose of opioid or stop using it altogether in order to relieve constipation, but worsening the burden that they already experience with pain [19]. In this regard, a large European survey has indicated that $40 \%$ of patients reduce their opioid intake due to OIC [20]. Moreover, both weakand strong-opioid users suffer comparable bother and decreased QoL $[15,19,20]$. The failure to achieve a balance between control of pain and OIC thus impairs QoL and compromises effective management of pain, limiting work productivity and reducing health-related QoL [21].

The results of a Delphi survey involving 190 palliative care specialists appear to indicate that there is a high level of consensus regarding pain management [22]. However, better understanding of OIC may aid in monitoring and treating OIC. In recent years, in many areas of medicine, there has been increased recognition that not only the disease, but also the therapy used to treat it, has a major impact on the patient's QoL. This has led to increased use of patient-reported outcome measures (PROMs), which reflect the outcomes of intervention from the subjective point of view of the patient. This is important, since the burden associated with the experience of treatment can approach that of the disease itself. PROMs are typically evaluated using standardized, self-administered questionnaires, and probe domains such as QoL, symptoms, mental health, and ability to perform activities related to daily living [23].

In the attempt to better understand the impact of OIC and improve its management, we carried out a survey of patients being treated with opioids. The overall objective of this survey was to analyze the impact of OIC on pain treatments and patient QoL. Towards this end, we examined the impact and consequences of OIC on the patient's life, relief of pain, and adherence to therapy. Patients with cancer-related and non-cancer pain were studied.

\section{METHODS}

\section{Study Design}

Online surveys were conducted by Insight Dojo, Guildford, UK, which used previously described panels used for market research in which respondents were invited to take part independently of this study [20]. Panelists were recruited to the online survey via invitation only. These online databases/panels contain no information about the patient's residence or what hospital they attend. Moreover, being anonymous panels used for market research, there is no specific sponsor or clinician recommending that patients participate in the study; rather patients are recruited from the online panel of which they are a member, based on extensive profiling. The panelists undergo frequent quality checks for average survey completion time, verbatim data, logic checks, and cross checks against profiled information in a survey (any fraudulent responders are removed and replaced), and repeat offenders are permanently removed from the panel. Patients from this panel were invited to take part in the study based on information held on their profile. Patients are asked to complete a short "screener" with questions relating to age, gender, pain medication taken and the pain condition they 
suffer from, and treated with opioids. Patients qualified for the survey if they suffered from one of the following conditions: psoriatic arthritis, rheumatoid arthritis, osteoarthritis, joint pain, chronic back pain, or pain relating to cancer, and were being treated either with a "weak" or "strong" opioid. Participants agreeing to take part in the survey did so by completing an Internet-based survey. The criteria used to determine the presence of OIC were as follows: new or worsening constipation when initiating, changing, or increasing opioid treatment, and either fewer than two bowel movements a week or reporting type 1 or 2 on the Bristol Stool Scale in the past week.

Data were stored in a secure database protected by username and password. The survey was carried out from January 2020 to February 2020. All data were anonymized, and we never received any personal data from the respondents. For this reason, the privacy of the patients has been protected throughout, as per Italian Privacy Authority regulation on the protection of personal data. Ethics committee approval was not required for this study. In Italy, survey research is not among studies that require the approval or written consent of research ethics committees (RECs); as previously indicated, respect of patient privacy is the only requirement for this type of survey, according to Italian law, as per Legislative Decree no. 196 of 30 June 2003, amended by Legislative Decree No. 101 of 10 August 2018, and Regulation (EU) 2016/679. Enrolled participants were informed about the details of the research and the potential to publish the results. All signed an informed consent form for both activities.

Patients were shown a list of opioids and were asked to state whether they currently took one of the products listed. Strong opioids included buprenorphine, fentanyl, hydromorphone, methadone, morphine, oxycodone and tapentadol. Weak opioids included codeine and tramadol. Patients were not asked which specific opioid they were taking, because it was not one of the objectives of the study.

\section{Study Questionnaire}

The questionnaire assessed five main areas: (i) general QoL and impact of pain (using PROMIS validated scales); (ii) impact of OIC (using the Patient Assessment of ConstipationSymptoms [PAC-SYM] and Patient Assessment of Constipation-Quality of Life [PAC-QOL] validated scales); (iii) risk factors for OIC; (iv) opioid and OIC management; (v) the patient journey. Opioids were categorized according to whether they were weak or equipotent/strong relative to morphine. PROMIS ${ }^{\circledR}$ (Patient-Reported Outcomes Measurement Information System) is a set of person-centered measures that evaluates and monitors physical, mental, and social health in adults and children. It can be used with the general population and with individuals living with chronic conditions [24]. The PAC questionnaires were used to measure specific symptoms (PAC-SYM) and QoL outcomes (PAC-QOL) in patients with constipation [25].

\section{Statistical Analysis}

The statistical approach combined both descriptive analyses and statistical comparisons. For continuous variables, quantitative descriptive statistics were calculated, including mean, range and standard deviation (SD). Categorical variables were summarized as counts and percentages. Comparison of different variables was performed with $\mathrm{Z}$ tests in the case of quantitative variables where statistics within different subgroups were compared.

\section{RESULTS}

\section{Patient Characteristics}

A total of 597 patients completed the online survey, $150(25 \%)$ with cancer-related pain and 447 (75\%) with non-cancer pain. In addition, 201 (34\%) patients reported no OIC, and 396 (66\%) reported OIC; $78 \%$ of patients were $<60$ years of age, with an opioid therapy period that 
Table 1 Characteristics of respondents

\begin{tabular}{|c|c|c|c|}
\hline & $\begin{array}{l}\text { Total } \\
N=\mathbf{5 9 7} \\
n(\%)\end{array}$ & $\begin{array}{l}\text { Cancer pain } \\
N=150 \\
n(\%)\end{array}$ & $\begin{array}{l}\text { Non-cancer pain } \\
N=447 \\
n(\%)\end{array}$ \\
\hline \multicolumn{4}{|l|}{ Gender } \\
\hline Male & $314(53 \%)$ & $68(45 \%)$ & $246(55 \%)$ \\
\hline Female & $283(47 \%)$ & $82(55 \%)$ & $201(45 \%)$ \\
\hline \multicolumn{4}{|l|}{ Age (years) } \\
\hline $40-49$ & $223(37 \%)$ & $52(35 \%)$ & $171(38 \%)$ \\
\hline $50-59$ & 247 (41\%) & 89 (59\%) & $158(35 \%)$ \\
\hline $60-69$ & $97(16 \%)$ & $7(5 \%)$ & $90(20 \%)$ \\
\hline $70-79$ & $28(5 \%)$ & $2(1 \%)$ & $26(6 \%)$ \\
\hline$\geq 80$ & $2(0.3 \%)$ & $0(0 \%)$ & $2(0.4 \%)$ \\
\hline \multicolumn{4}{|l|}{ Current opioid } \\
\hline Strong & $386(65 \%)$ & $139(93 \%)$ & $247(55 \%)$ \\
\hline Weak & $506(85 \%)$ & $131(87 \%)$ & $375(84 \%)$ \\
\hline \multicolumn{4}{|c|}{ Duration of opioid therapy } \\
\hline $1-3$ months & $77(13 \%)$ & $17(11 \%)$ & $60(13 \%)$ \\
\hline$>3$ months $-<1$ year & $156(26 \%)$ & $20(20 \%)$ & $126(28.2 \%)$ \\
\hline $1-3$ years & $207(35 \%)$ & $84(56 \%)$ & $123(28 \%)$ \\
\hline $3-5$ years & $100(17 \%)$ & $15(10 \%)$ & $85(19 \%)$ \\
\hline $5-10$ years & $38(6 \%)$ & $4(3 \%)$ & $34(8 \%)$ \\
\hline$>10$ years & $19(3 \%)$ & $0(0 \%)$ & $19(4 \%)$ \\
\hline \multicolumn{4}{|l|}{ OIC } \\
\hline Yes & $396(66 \%)$ & $100(67 \%)$ & $296(66 \%)$ \\
\hline No & $201(34 \%)$ & $50(33 \%)$ & $151(34 \%)$ \\
\hline
\end{tabular}

was $<1$ year in $39 \%$ and $>3$ years in $26 \%$ (Table 1).

\section{General QoL and Impact of Pain}

PROMIS global physical and mental health scores and PROMIS pain interference scores are shown in Fig. 1. Patients with cancer pain had somewhat lower physical and mental health scores overall than non-cancer patients. The presence of OIC had an impact on mental health in both cancer and non-cancer patients but had little effect on physical health. An analysis of a subset of PROMIS pain interference questions indicated that pain is more likely to interfere with a patient's life when they have OIC (Fig. 1).

\section{Impact of OIC}

Both PAC-QOL and PAC-SYM were higher among cancer patients, with opioid strength 
having some effect on PAC-SYM among the group with chronic non-cancer pain (Fig. 2). Compared to patients with chronic pain and OIC, those with cancer pain and OIC reported a greater impact on QoL.

The majority of OIC patients with both cancer and non-cancer pain experienced only pain-related symptoms or only bowel movement-related symptoms (Fig. 3). In contrast, only $19 \%$ of chronic patients reported symptoms that involved both pain and bowel movements. A small proportion of patients with cancer or non-cancer pain reported that the symptoms involved only bowel movement-related symptoms, and very few reported that their symptoms were related to pain alone (Fig. 3).

\section{Risk Factors for OIC}

In both cancer and non-cancer patients, younger age and less time on opioids were

\section{PROMIS Global physical health score}

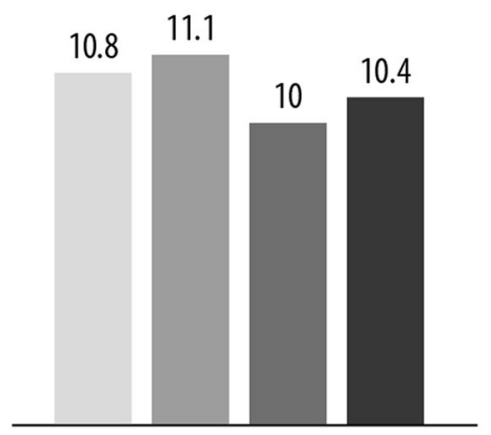

PROMIS Global mental health score

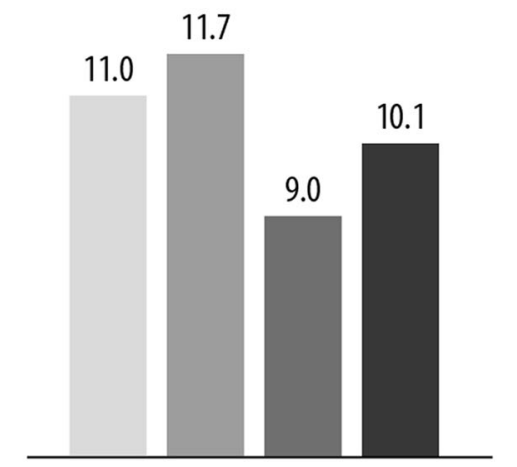

PROMIS Pain interference score sum (SUBSET)

\section{$\square$ Chronic pain with OIC $\square$ Chronic pain without OIC \\ - Cancer pain with OIC $\quad$ Cancer pain without OIC}

Fig. 1 PROMIS global physical health, mental health, and pain interference scores. For global physical health and mental health, a higher score indicated better health, while for pain interference a higher score indicated more interference

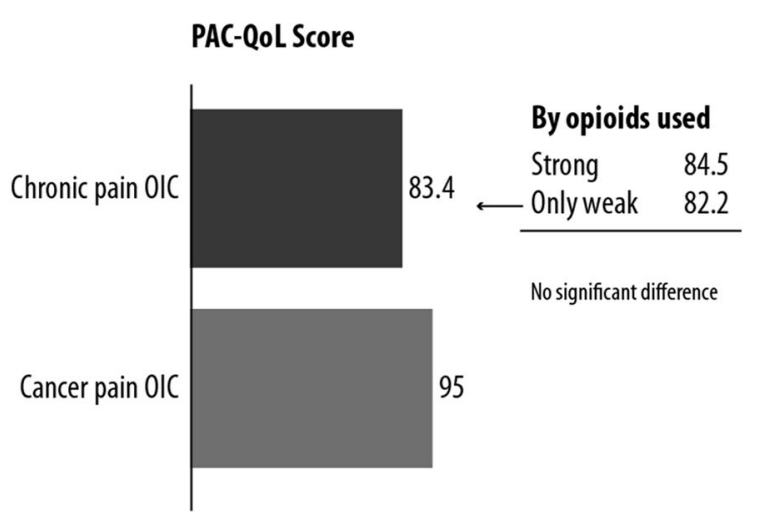

Fig. 2 PAC-SYM and PAC-QoL scores in patients with chronic non-cancer pain and cancer pain. A higher score indicates greater impact. The sample size was too small for analysis by strong vs. weak opioids in cancer pain since
PAC-SYM Score

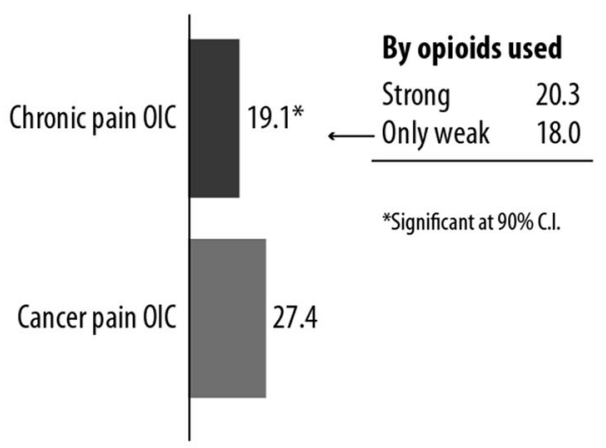

most patients were receiving strong opioids. There was no significant difference in PAC-QoL score between chronic pain and cancer pain 


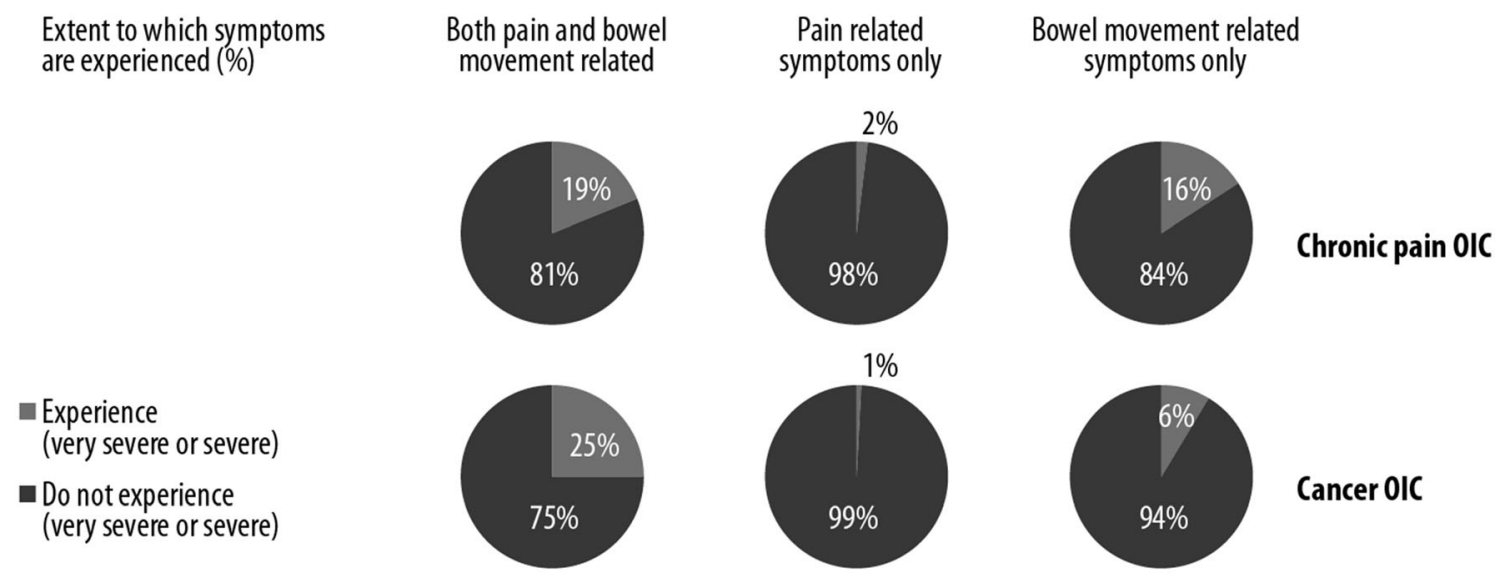

Fig. 3 OIC in patients with cancer $(n=100)$ or chronic pain $(n=296)$

Table 2 Correlations between OIC and PAC-QoL factors

\begin{tabular}{lll}
\hline Factor & Cancer pain & Non-cancer pain \\
\hline Younger age & $0.209^{* *}$ & $0.188^{* *}$ \\
Less time on opioids & $0.201^{*}$ & $0.131^{*}$ \\
OIC symptoms & & \\
Painful bowel movements & $0.655^{* *}$ & $0.634^{* *}$ \\
Abdominal pain & $0.663^{* *}$ & $0.564^{* *}$ \\
Incomplete bowel movement & $0.564^{* *}$ & $0.576^{* *}$ \\
Abdominal bloating & $0.520^{* *}$ & $0.539^{* *}$ \\
Difficulty in bowel movement & $0.436^{* *}$ & $0.521^{* *}$ \\
\hline
\end{tabular}

Correlation coefficients are shown

*Significant at $P=0.05$ (2-tailed)

${ }^{* *}$ Significant at $P=0.01$ (2-tailed)

significantly associated with a greater impact of OIC on QoL (Table 2). Specific OIC symptoms were also significantly correlated with poorer QoL.

\section{Opioids and OIC Management}

Most cancer patients manage their constipation with laxatives (65\%), compared to only a third of those with non-cancer pain (34\%) (Fig. 4). In patients with non-cancer pain, the most common methods to reduce constipation involved drinking more fluids and making dietary changes; $34 \%$ said that they used laxatives, and $21 \%$ said they used more than one. In patients with cancer pain, 65\% said they were using laxatives (Fig. 4). Other techniques were used in smaller proportions of patients in both groups. Overall, $41 \%$ of patients with non-cancer pain were satisfied with the current means of constipation management, while $22 \%$ were dissatisfied and $37 \%$ were neither satisfied nor dissatisfied (Fig. 5). Moreover, 22\% of patients with non-cancer pain were dissatisfied with constipation management techniques compared to $34 \%$ of cancer patients (Fig. 5). 


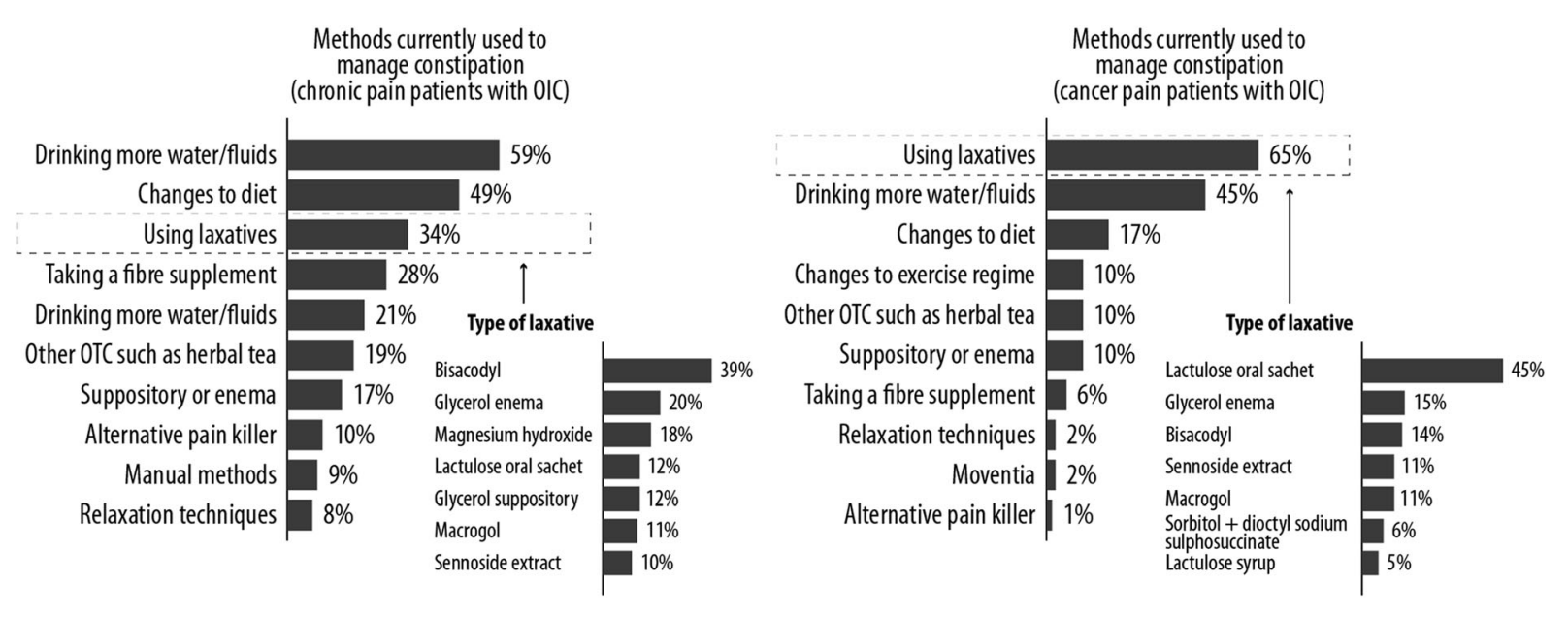

Fig. 4 Methods used to manage constipation in chronic or cancer patients

\section{A} Satisfaction with your current
means of constipation
management in terms of how
much it helps you to improve
your bowel movements

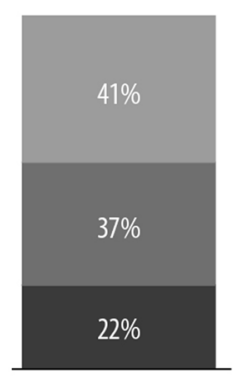

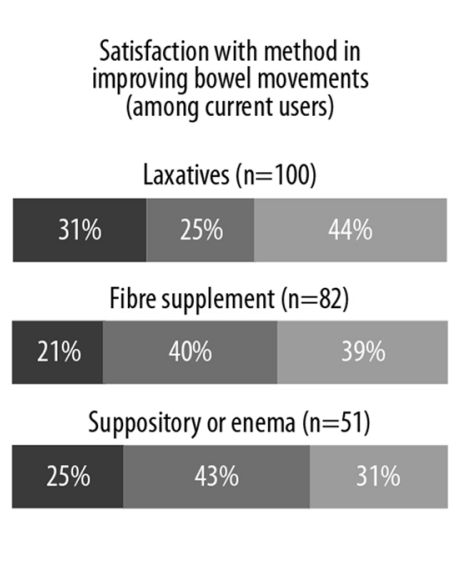

- Dissatisfied $\quad$ Neither satisfied nor dissatisfied $\quad$ Satisfied
Satisfaction with your current means of constipation management in terms of how much it helps you to improve your bowel movements
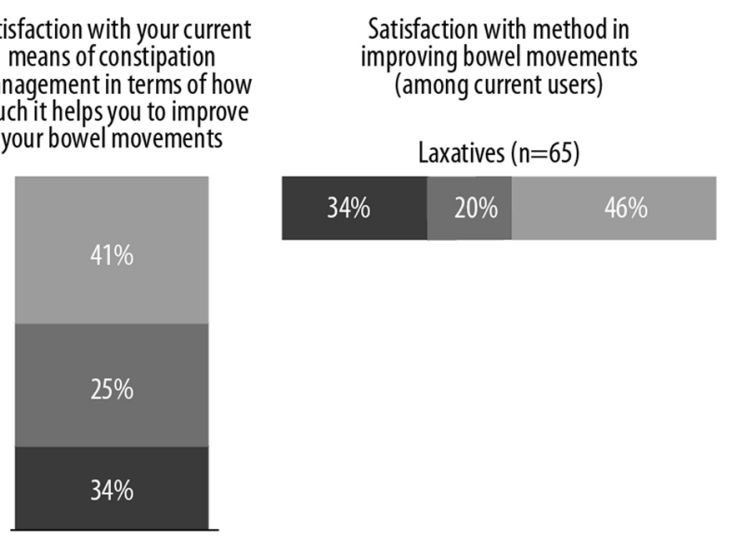

Fig. 5 Satisfaction with constipation management techniques. Patients with non-cancer pain (a). Patients with cancer pain (b)

Many patients felt that constipation made adherence to their opioid regimen more difficult and that pain relief had become less effective (Fig. 6). In patients with cancer, 67\% reported that it was a little or a lot more difficult to adhere to their opioid regimen, compared to $46 \%$ of non-cancer patients. Moreover, $61 \%$ of cancer patients said that the pain relief was a little less or significantly less effective after experiencing constipation, compared to $28 \%$ of those with non-cancer pain.

Over half of those with non-cancer pain said that they interrupted their opioid regimen due to their constipation, compared to only $6 \%$ of those with cancer pain (Fig. 7). Patients with cancer used a variety of methods to change their opioid therapy. The most common were skipping a dose (30\%), taking a short break (30\%), and taking less than the prescribed dose or frequency (27\%).

Constipation had been discussed with their healthcare provider (HCP) in $48 \%$ of non-cancer patients and in $73 \%$ of cancer patients. In addition, a prescription for OIC had been given to $30 \%$ of those with non-cancer pain and $41 \%$ of those with cancer pain. 
To what extent has constipation affected your ability to adhere to your opioid regimen?

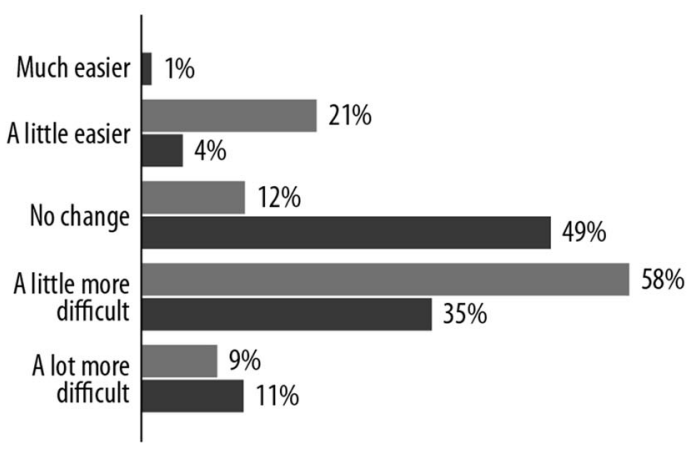

What changes, if any, have you noticed in the pain relief you are getting from your opioid regimen since you began experiencing constipation?

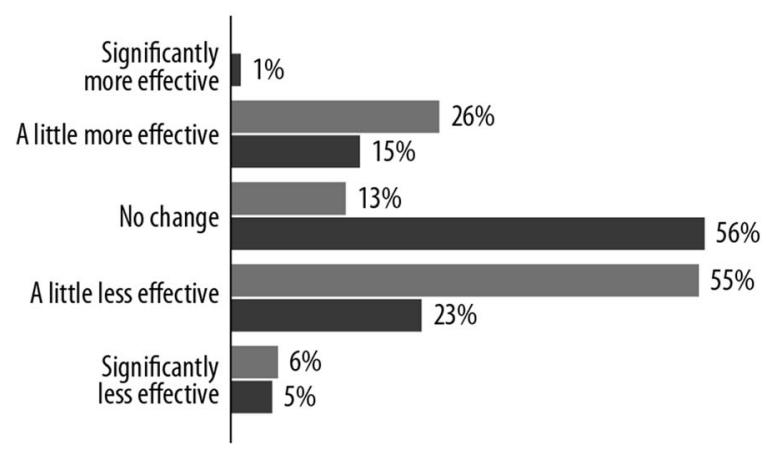

- Chronic pain with OIC $\quad$ Cancer pain with OIC

Fig. 6 Extent to which constipation affects ability to adhere to opioid regime and changes in pain relief after experiencing constipation
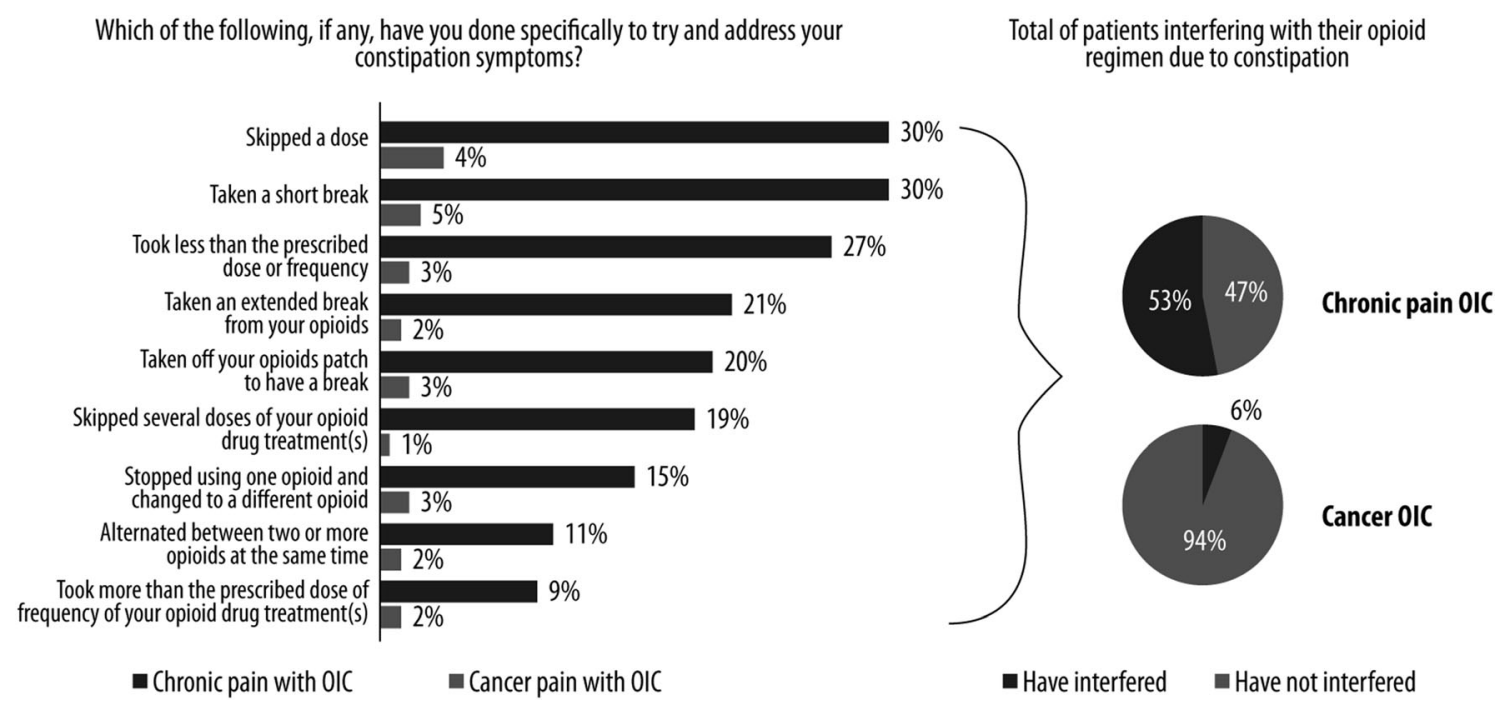

Fig. 7 Changes to opioid regimen in patients with OIC

\section{Patient Journey}

More patients with cancer pain are dissatisfied with how the healthcare system managed their OIC (Fig. 8). In those with chronic pain and OIC, 24\% expressed varying degrees of dissatisfaction, compared to $37 \%$ in those with cancer pain and OIC. Among all patients, satisfaction with the healthcare system was higher among those who had spoken to an HCP about their constipation ( $45 \%$ vs. $17 \%$ satisfied). Patients with OIC who were not warned about constipation at first prescription were more likely to make changes to their opioid regimen (71\% not warned vs. 59\% warned). Among those who were warned about constipation at repeat prescription, $50 \%$ of patients were satisfied with their HCP, compared to $25 \%$ who were not warned.

Moreover, patients with OIC who were not warned about constipation were more likely to make changes to their opioid regimen. Among patients who were warned by their HCP about constipation at first prescription, 59\% made 
A Satisfaction with overall management of $0 \mid C$ by healthcare system

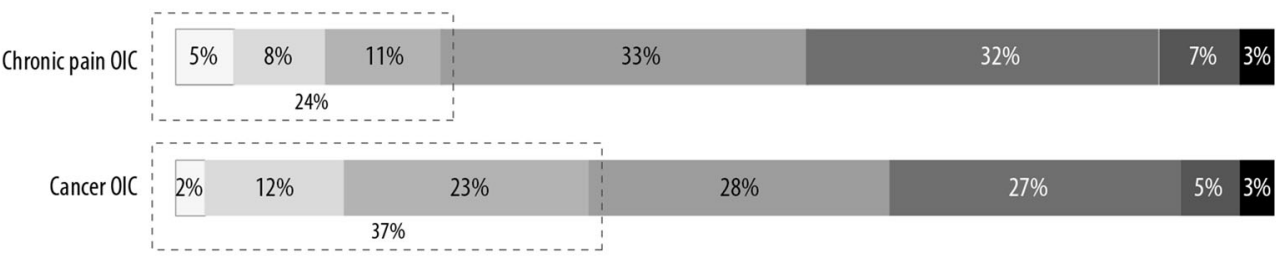

B

When have any conversation with HCP about constipation

When have never had a conversation with HCP about constipation

When are warned about constipation when opioids are first prescribed

When aren't
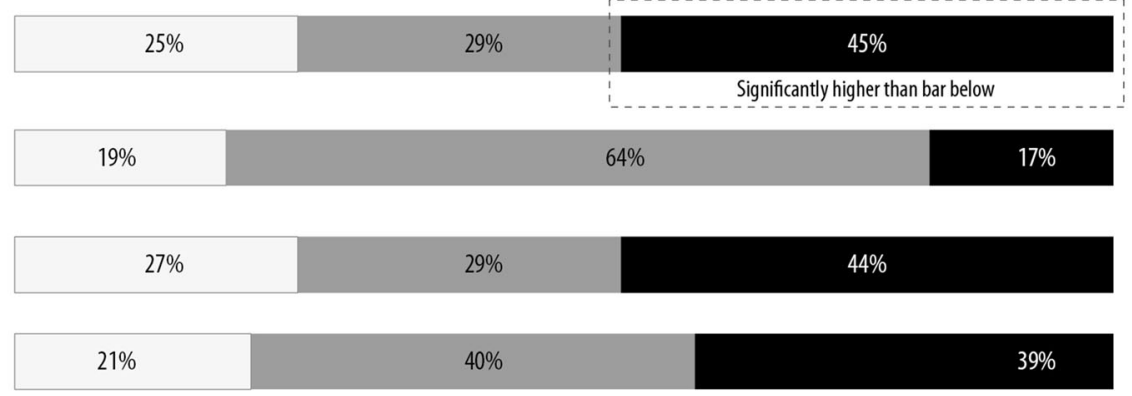

When are warned about constipation at repeat prescription

When aren't

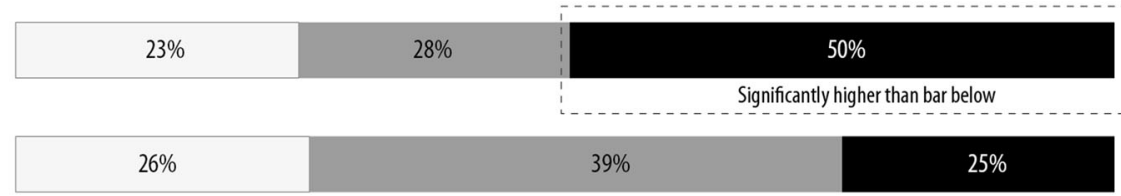

Have made a change to opioid regimen

C When have any conversation with HCP about constipation

$36 \%$

$64 \%$

When have never had a conversation with HCP about constipation

$31 \%$

$69 \%$

When are warned about constipation when opioids are first prescribed

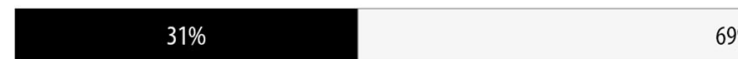

$41 \%$

$59 \%$

When aren't

\section{$29 \%$}

$71 \%$

When are warned about constipation at repeat prescription

When aren't

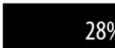

$28 \%$

$72 \%$

Significantly higher than bar below

$\begin{array}{rlcccc}\begin{aligned} \text { Completely } \\ \text { dissatisfied }\end{aligned} & \text { Mostly } & \text { dissatisfied } & \text { Somewhat } & \text { dissatisfied } & \text { Neither satisfied } \\ \text { not dissatisfied } & \begin{array}{c}\text { Somewhat } \\ \text { satisfied }\end{array} & \begin{array}{c}\text { Mostly } \\ \text { satisfied }\end{array} & \begin{array}{c}\text { Completely } \\ \text { satisfied }\end{array}\end{array}$

Fig. 8 Overall satisfaction with overall management of OIC by the healthcare system (a). Details about satisfaction in all patients with chronic pain (b). Changes made to opioid regimen in patients who were not warned about constipation $(\mathbf{c})$ 
changes to their opioid regimen, compared to $71 \%$ who were not warned. At repeat prescription, $56 \%$ of patients who were warned about constipation made changes to their opioid regimen, compared to $72 \%$ who were not.

\section{DISCUSSION}

The present survey demonstrates that OIC has a significant negative impact on QoL, affecting mental health more than physical health. While OIC has been reported to be a source of psychological distress [26], measurement of both mental and physical health has not been widely addressed. Bell et al. [27] reported that health-related QoL mental and physical component scores were reduced in patients with OIC compared to those without it. Our results highlight that further research is needed on the specific mental and physical burden of OIC.

The impact of OIC was felt by all patients with both strong and weak opioid therapy, but was particularly burdensome for patients with cancer-related pain. Many patients reported that management of OIC was unsatisfactory and that they had altered their opioid regimen in order to address constipation as a consequence. A substantial proportion of patients were also dissatisfied with the overall management of their OIC. Dissatisfaction was reported by $31 \%$ on laxatives, $21 \%$ on fiber supplementation, and $25 \%$ of those using a suppository or enema. Around one-third of patients were also actively dissatisfied with the support provided by the healthcare system, signaling that there is room for improvement. This is further exacerbated when providers do not speak to patients about constipation, which leads to additional non-compliance.

Unlike most studies on OIC, we included patients with both cancer-related and noncancer pain, and differences in the impact of OIC in these two groups were seen in some instances. Firstly, a higher proportion of those with cancer-related pain reported at least one "severe" or "very severe" constipation symptom compared to those with cancer-related pain. Moreover, $75 \%$ of cancer patients with OIC reported the presence of severe pain and bowel movement-related symptoms, compared to non-cancer patients. Moreover, almost twice as many cancer patients used laxatives, and more than one half said that they altered their opioid regimen due to constipation. These results help explain why more cancer patients were dissatisfied with the management of their OIC than those with non-cancer pain.

Our findings on QoL further confirm previous surveys of patients with OIC. For example, Christensen reported that a large proportion of patients with OIC have moderate to very severe abdominal symptoms, together with low satisfaction with laxatives and low health-related QoL [13]. In a previous survey on patients with non-cancer pain, other authors also found that laxatives were associated with a low rate of satisfactory response [28]. In a survey of 184 patients taking opioids for at least one month in the UK, the use of laxatives to treat opioid-induced constipation was reported to be frequently ineffective [29]. Moreover, instead of providing relief from OIC, laxatives often led to side effects such as bloating [29]. Our results add additional weight to these previous findings that laxatives are not completely effective in managing OIC. Of note, the present survey was carried out before the reimbursement approval of methylnaltrexone bromide, naloxegol, and naldemedine in Italy, and thus it would be of interest to determine the impact of these agents on OIC.

A survey on almost 19,000 participants reported that both weak- and strong-opioid users suffer from comparable bother and decreased QoL, and that about one in five patients is dissatisfied with treatment of OIC [20]. We also found that the impact of a strong or weak opioid in PAC-SYM and PAC-QoL scores was similar in patients with either non-cancer pain or cancer pain. Another important aspect reported by almost half of patients was the lack of counseling about constipation. Similar to the results seen herein, in a previous survey more than half of patients were not adequately counseled about constipation, and as a consequence altered their opioid regimen to compensate for OIC [20]. Along these lines, a retrospective analysis of 216 patient-provider discussions in the United States also found that 
HCP counseling and management of OIC was inadequate, and that in around 30\% of cases no specific treatments or guidance was given [30]. Our findings mirror those results, since a prescription for OIC was given in only around onethird of cases.

The lack of adequate counseling by HCPs may be due to the difference in perceptions of OIC by HCPs and patients. In this regard, other authors reported that HCPs did not fully appreciate the symptoms of OIC or the significant impact that OIC has on management of pain and the patient's QoL [28]. A previous study also noted that while HCPs may acknowledge that OIC is a concern, they tend to prioritize management of pain over constipation [31]. This highlights the need for better communication between patients and HCPs, and for improved HCP counseling about OIC whenever an opioid is initiated. An analysis of HCPs, patients, and caregivers stressed that both pharmacological and non-pharmacological approaches need to be considered and their use facilitated by HCPs [32]. An analysis of patient responses on OIC further reported that patients are indeed motivated to speak to their HCP about OIC [33]. HCPs thus need to be more engaged in actively managing OIC.

Considering that OIC is not effectively managed despite a number of treatment options, a European expert consensus statement has given indications for a stepwise approach for clinicians [34]. When constipation is clearly related to initiation, dose escalation, or switching of opioid treatment, laxatives as first-line treatment and PAMORA or alternative opioid antagonists as-second line should be given, and the patient should be referred to a specialist in the use of opioids. The same group of experts also noted that lack of awareness among clinicians about OIC in patients on opioid therapy represents a barrier to adequate pain management. Moreover, even if they are aware of OIC, HCPs may not query patients about OIC.

The main limitations of this survey are the low proportion of cancer patients (25\%) and the lack of information on other treatments or clinical aspects that can cause constipation, and therefore the onset of OIC. Moreover, constipation in cancer patients may be related to various treatments and/or cancer-related, which was not addressed herein. In any case, this survey can be considered to be representative of clinical practice in Italy.

\section{CONCLUSION}

Our results show that management of OIC is inadequate in many cases, and thus indicate a definite need for education of HCPs about OIC and its negative consequences. Furthermore, to educate patients about OIC, when initiating an opioid prescription, HCPs should be encouraged to actively ask patients about OIC at every follow-up visit. Patients should also be asked about possible alterations in the opioid regimen, which we found to be particularly common among patients with cancer-related pain.

Overcoming barriers to adequate management of OIC thus provides the opportunity to improve the patient's QoL and lessen functional impairment [34]. In this regard, it has been recently stressed that HCPs should adequately inform patients about the possibility of OIC and that this aspect needs to be carefully addressed in management guidelines $[35,36]$. This is especially relevant given that there are now a number of effective treatment options, such as PAMORAs, which are now part of a newly proposed algorithm for management of OIC.

\section{ACKNOWLEDGEMENTS}

The authors thank the participants of the study.

Funding. This study and the journal's Rapid Service Fee has been supported by the Paolo Procacci Foundation.

Medical Writing Assistance. Editorial assistance in drafting the manuscript was provided by Dr. Patrick Moore, medical writer, on behalf of Content Ed Net. Support for this assistance was funded by Molteni and C Dei Fratelli Alitti and Shionogi Srl, Italy.

Authorship. All named authors meet the International Committee of Medical Journal 
Editors (ICMJE) criteria for authorship for this article, take responsibility for the integrity of the work as a whole, and have given their approval for this version to be published.

Authors' Contributions. Giustino Varrassi, Walter Gianni, Franco Marinangeli and Carmine Pinto contributed to the final paper revising the manuscript, stating the discussion and conclusion; Vivek Banerji has had in charge survey design and analysis. All the authors have reviewed the final manuscript and accepted it for submission.

Disclosures. Giustino Varrassi is President of the Paolo Procacci Foundation. He is also Editor-in-Chief for Pain and Therapy. Vivek Banerji is Insight Dojo employee. Walter Gianni, Franco Marinangeli, Carmine Pinto declare that they have no potential conflict of interest.

Compliance with Ethics Guidelines. All data were anonymized, and we never received any personal data from the respondents. For this reason, the privacy of the patients has been protected throughout, as per Italian Privacy Authority regulation on the protection of personal data. Ethics committee approval was not required for this study. In Italy, survey research is not among studies that require the approval or written consent of Research Ethics Committees (RECs). Informed consent was distributed to every enrolled patient. Patients were aware that collected survey data should be published.

Data Availability. The datasets generated during and/or analyzed during the current study are available from the corresponding author on reasonable request.

Open Access. This article is licensed under a Creative Commons Attribution-NonCommercial 4.0 International License, which permits any non-commercial use, sharing, adaptation, distribution and reproduction in any medium or format, as long as you give appropriate credit to the original author(s) and the source, provide a link to the Creative Commons licence, and indicate if changes were made. The images or other third party material in this article are included in the article's Creative Commons licence, unless indicated otherwise in a credit line to the material. If material is not included in the article's Creative Commons licence and your intended use is not permitted by statutory regulation or exceeds the permitted use, you will need to obtain permission directly from the copyright holder. To view a copy of this licence, visit http://creativecommons.org/licenses/by$\mathrm{nc} / 4.0 /$.

\section{REFERENCES}

1. Hauser W, Morlion B, Vowles KE, et al. European* clinical practice recommendations on opioids for chronic noncancer pain-Part 1: role of opioids in the management of chronic noncancer pain. Eur J Pain. 2021;25(5):949-68. https://doi.org/10.1002/ ejp.1736.

2. O'Brien T, Christrup LL, Drewes AM, et al. European Pain Federation position paper on appropriate opioid use in chronic pain management. Eur J Pain. 2017;21:3-19.

3. Camilloni A, Nati G, Maggiolini P, et al. Chronic non-cancer pain in primary care: an Italian crosssectional study. Signa Vitae. 2021;17(2):54-62. https://doi.org/10.22514/sv.2020.16.0111.

4. Del Giorno R, Frumento P, Varrassi G, et al. Assessment of chronic pain and access to pain therapy: a cross-sectional population-based study. J Pain Res. 2017;10:2577-84.

5. Latina R, De Marinis MG, Giordano F, et al. Epidemiology of chronic pain in the Latium region, Italy: a cross-sectional study on the clinical characteristics of patients attending pain clinics. Pain Manag Nurs. 2019;20:373-81.

6. Bialas P, Maier C, Klose P, et al. Efficacy and harms of long-term opioid therapy in chronic non-cancer pain: Systematic review and meta-analysis of openlabel extension trials with a study duration $>/=26$ weeks. Eur J Pain. 2020;24:265-78.

7. Meske DS, Lawal OD, Elder H, et al. Efficacy of opioids versus placebo in chronic pain: a systematic review and meta-analysis of enriched enrollment randomized withdrawal trials. J Pain Res. 2018;11: 923-34.

8. Camilleri M, Drossman DA, Becker G, et al. Emerging treatments in neurogastroenterology: a multidisciplinary working group consensus 
statement on opioid-induced constipation. Neurogastroenterol Motil. 2014;26:1386-95.

9. Webster LR. Opioid-induced constipation. Pain Med. 2015;16(Suppl 1):S16-21.

10. Kalso E, Edwards JE, Moore RA, et al. Opioids in chronic non-cancer pain: systematic review of efficacy and safety. Pain. 2004;112:372-80.

11. Abramowitz L, Beziaud N, Labreze L, et al. Prevalence and impact of constipation and bowel dysfunction induced by strong opioids: a crosssectional survey of 520 patients with cancer pain: DYONISOS study. J Med Econ. 2013;16:1423-33.

12. Pappagallo M. Incidence, prevalence, and management of opioid bowel dysfunction. Am J Surg. 2001;182:11S-S18.

13. Christensen HN, Olsson U, From J, et al. Opioidinduced constipation, use of laxatives, and healthrelated quality of life. Scand J Pain. 2016;11:104-10.

14. Viscusi ER. Clinical overview and considerations for the management of opioid-induced constipation in patients with chronic noncancer pain. Clin J Pain. 2019;35:174-88.

15. Holzer P, Ahmedzai SH, Niederle N, et al. Opioidinduced bowel dysfunction in cancer-related pain: causes, consequences, and a novel approach for its management. J Opioid Manag. 2009;5:145-51.

16. Kumar L, Barker C, Emmanuel A. Opioid-induced constipation: pathophysiology, clinical consequences, and management. Gastroenterol Res Pract. 2014;2014:141737.

17. Coluzzi F, Scerpa MS, Pergolizzi J. Naldemedine: a new option for OIBD. J Pain Res. 2020;13:1209-22.

18. AIFA. http://www.agenziafarmaco.gov.it/content/ nota-90. Accessed 27 Oct 2020.

19. Bell TJ, Panchal SJ, Miaskowski C, et al. The prevalence, severity, and impact of opioid-induced bowel dysfunction: results of a US and European Patient Survey (PROBE 1). Pain Med. 2009;10:35-42.

20. Andresen V, Banerji V, Hall G, et al. The patient burden of opioid-induced constipation: New insights from a large, multinational survey in five European countries. United European Gastroenterol J. 2018;6:1254-66.

21. Coyne KS, LoCasale RJ, Datto CJ, et al. Opioid-induced constipation in patients with chronic noncancer pain in the USA, Canada, Germany, and the UK: descriptive analysis of baseline patient-reported outcomes and retrospective chart review. Clinicoecon Outcomes Res. 2014;6:269-81.
22. Varrassi G, De Conno F, Orsi L, et al. Cancer Pain Management: An Italian Delphi Survey from the Rational Use of Analgesics (RUA) Group. J Pain Res. 2020;13:979-86.

23. Kingsley C, Patel S. Patient-reported outcome measures and patient-reported experience measures. BJA Educ. 2017;17:137-44.

24. PROMIS. https://www.healthmeasures.net/exploremeasurement-systems/promis. Accessed 19 Oct 2020.

25. Mapi Research Trust. https://eprovide.mapi-trust. org/instruments/patient-assessment-of-constipationsymptoms. Accessed 19 Oct 2020.

26. Dhingra L, Shuk E, Grossman B, et al. A qualitative study to explore psychological distress and illness burden associated with opioid-induced constipation in cancer patients with advanced disease. Palliat Med. 2013;27:447-56.

27. Bell T, Annunziata K, Leslie JB. Opioid-induced constipation negatively impacts pain management, productivity, and health-related quality of life: findings from the National Health and Wellness Survey. J Opioid Manag. 2009;5:137-44.

28. LoCasale RJ, Datto C, Margolis MK, et al. Satisfaction with therapy among patients with chronic noncancer pain with opioid-induced constipation. J Manag Care Spec Pharm. 2016;22:246-53.

29. Emmanuel A, Johnson M, McSkimming P, et al. Laxatives do not improve symptoms of opioid-induced constipation: results of a patient survey. Pain Med. 2017;18:1932-40.

30. Vallerand AH, Hendry S, Baldys E, et al. Analysis of patient-provider interactions regarding the burden and treatment of opioid-induced constipation in adults with chronic noncancer pain. Pain Med. 2019;20:889-96.

31. Keller MS, Jusufagic A, Spiegel BMR. Patient and provider differences in the treatment of opioid-induced constipation: a qualitative study. BMC Gastroenterol. 2019;19:182.

32. Hasson F, Muldrew D, Carduff E, et al. "Take more laxatives was their answer to everything": A qualitative exploration of the patient, carer and healthcare professional experience of constipation in specialist palliative care. Palliat Med. 2020;34: 1057-66.

33. Epstein RS, Teagarden JR, Cimen A, et al. When people with opioid-induced constipation speak: a patient survey. Adv Ther. 2017;34:725-31. 
34. Farmer AD, Drewes AM, Chiarioni G, et al. Pathophysiology and management of opioid-induced constipation: European expert consensus statement. United European Gastroenterol J. 2019;7: $7-20$.

35. Alvaro D, Caraceni AT, Coluzzi F, et al. What to do and what not to do in the management of opioidinduced constipation: a choosing wisely report.
Pain Ther. 2020;9:657-67. https://doi.org/10.1007/ s40122-020-00195-z.

36. Pergolizzi JV Jr, Christo PJ, LeQuang JA, et al. The use of peripheral mu-opioid receptor antagonists (PAMORA) in the management of opioid-induced constipation: an update on their efficacy and safety. Drug Des Devel Ther. 2020;14:1009-25. 